TRANSACTIONS OF THE

AMERICAN MATHEMATICAL SOCIETY

Volume 353, Number 1, Pages 157-173

S 0002-9947(00)02642-8

Article electronically published on June 20, 2000

\title{
ENDOFINITENESS IN STABLE HOMOTOPY THEORY
}

\author{
HENNING KRAUSE AND ULRIKE REICHENBACH
}

\begin{abstract}
We study endofinite objects in a compactly generated triangulated category in terms of ideals in the category of compact objects. Our results apply in particular to the stable homotopy category. This leads, for example, to a new interpretation of stable splittings for classifying spaces of finite groups.
\end{abstract}

\section{INTRODUCTION}

A CW-complex is said to be endofinite if all stable homotopy groups $\pi_{n}^{s}(X)=$ $\left\{S^{n}, X\right\}$ are finite length modules over the ring $\{X, X\}$ of stable self-maps of $X$. In this paper we study $\mathrm{CW}$-complexes having this finiteness property. One motivation for this work is to understand stable splittings of certain spaces, for instance the classifying space $B G$ of a finite group $G$. We shall give an algebraic interpretation of such splittings in terms of certain ideals in the category of finite spectra.

A natural framework for the study of endofinite objects is compactly generated triangulated categories. Thus we consider a triangulated category $\mathcal{C}$, for example the stable homotopy category, which has arbitrary coproducts and is generated by a set of compact objects (an object $X$ in $\mathcal{C}$ is compact if the representable functor $\operatorname{Hom}(X,-)$ preserves coproducts). An object $X$ in $\mathcal{C}$ is said to be endofinite if the group $\operatorname{Hom}(C, X)$ is a finite length module over the endomorphism $\operatorname{ring} \operatorname{End}(X)$ for all compact objects $C$ in $\mathcal{C}$. This finiteness condition has a number of interesting consequences. For example, in [15] it is shown that every endofinite object decomposes essentially uniquely into indecomposable objects with local endomorphism rings. Here, we shall study endofinite objects in terms of certain ideals in the full subcategory $\mathcal{C}_{0}$ of compact objects in $\mathcal{C}$. Viewing $\mathcal{C}_{0}$ as a ring with several objects, we consider for every $X$ in $\mathcal{C}$ the annihilator $\operatorname{Ann} X$ of $X$, which is the ideal of maps $\phi$ in $\mathcal{C}_{0}$ such that $\operatorname{Hom}(\phi, X)=0$. Two types of ideals $\mathfrak{I}$ in $\mathcal{C}_{0}$ are of particular importance. For the purpose of this introduction let us give an elementary definition; a conceptual description of these ideals can be found in Sections 1.2 and 1.3.

- $\mathfrak{I}$ is cohomological if for every sequence of maps in $\mathcal{C}_{0}$

$$
X^{\prime} \stackrel{\phi^{\prime}}{\longrightarrow} X \stackrel{\phi}{\longrightarrow} Y \stackrel{\phi^{\prime \prime}}{\longrightarrow} Y^{\prime \prime}
$$

such that $\phi^{\prime}$ is a fibre of $\phi^{\prime \prime} \phi$, the map $\phi$ belongs to $\mathfrak{I}$ if $\phi \phi^{\prime}$ and $\phi^{\prime \prime} \phi$ belong to $\mathfrak{I}$. We shall see that the cohomological ideals in $\mathcal{C}_{0}$ are precisely the annihilator ideals of objects in $\mathcal{C}$. In fact, for every cohomological ideal $\mathfrak{I}$ there exists a canonical object $E_{\mathfrak{I}}$ such that $\mathfrak{I}=\operatorname{Ann} E_{\mathfrak{I}}$.

Received by the editors November 18, 1998.

2000 Mathematics Subject Classification. Primary 55P42, 55 U35.

(C)2000 American Mathematical Society 
- $\mathfrak{I}$ is cofinite if for every $X$ in $\mathcal{C}_{0}$ there exists $n \in \mathbb{N}$ such that for every sequence of maps in $\mathcal{C}_{0}$

$$
X \stackrel{\phi_{0}}{\longrightarrow} X_{0} \stackrel{\phi_{1}}{\longrightarrow} X_{1} \stackrel{\phi_{2}}{\longrightarrow} \ldots \stackrel{\phi_{n}}{\longrightarrow} X_{n}
$$

the composition of $\phi_{i-1} \ldots \phi_{0}$ with a fibre of $\phi_{i} \ldots \phi_{0}$ belongs to $\mathfrak{I}$ for some $i \leq n$. Let us denote by $\operatorname{Max} \mathcal{C}_{0}$ the set of maximal elements among the cofinite cohomological ideals $\mathfrak{I} \neq \mathcal{C}_{0}$ in $\mathcal{C}_{0}$.

Theorem A. An object $X$ in $\mathcal{C}$ is endofinite if and only if the annihilator of $X$ is cofinite. Moreover the maps $X \mapsto \operatorname{Ann} X$ and $\mathfrak{I} \mapsto E_{\mathfrak{I}}$ induce mutually inverse bijections between the set of isomorphism classes of indecomposable endofinite objects in $\mathcal{C}$ and $\operatorname{Max} \mathcal{C}_{0}$.

Any endofinite object $X$ has an essentially unique decomposition $X \cong \coprod_{i \in I} X_{i}$ into indecomposable endofinite objects, and the annihilator of $X$ controls this decomposition. More precisely, an indecomposable object $Y$ arises as a direct factor of an endofinite object $X$ if and only if $\operatorname{Ann} X \subseteq \operatorname{Ann} Y$. This is a particular consequence of the following result:

Theorem B. Let $X$ be an endofinite object in $\mathcal{C}$ with $\mathfrak{I}=\operatorname{Ann} X$. Then

$$
\mathfrak{I}=\bigcap_{\mathfrak{I} \subseteq \mathfrak{J} \in \operatorname{Max} \mathcal{C}_{0}} \mathfrak{J},
$$

and $X$ has a unique decomposition

$$
X \cong \coprod_{\mathfrak{I} \subseteq \mathfrak{J} \in \operatorname{Max} \mathcal{C}_{0}} X_{\mathfrak{J}}
$$

such that each $X_{\mathfrak{J}}$ is a non-empty coproduct of copies of $E_{\mathfrak{J}}$. Moreover, Ann $X \subseteq$ Ann $Y$ holds for some object $Y$ if and only if $Y$ is isomorphic to a direct factor of a (co)product of copies of $X$.

Let us now explain some special results for the stable homotopy category of spectra. A fairly immediate consequence of Theorem B is the fact that we can work one prime at a time.

Corollary. Let $X$ be an endofinite spectrum. Then the $\mathfrak{p}$-localization map $X \rightarrow X_{\mathfrak{p}}$ is a split epimorphism for every prime ideal $\mathfrak{p}$ in $\mathbb{Z}$. If $X_{\text {tor }}$ denotes the fibre of the rational localization $X \rightarrow X_{(0)}$, then $X \cong X_{(0)} \vee X_{\text {tor }}$ and $X_{\text {tor }} \cong \bigvee_{\mathfrak{p}}\left(X_{\text {tor }}\right)_{\mathfrak{p}}$, where $\mathfrak{p}$ runs through all non-zero prime ideals in $\mathbb{Z}$.

Having developed a general theory of endofinite spectra, one may ask for conditions on a CW-complex $X$ such that its suspension spectrum is endofinite. It turns out that this happens if and only if all stable homotopy groups of $X$ are finite length modules over the ring of stable self-maps of $X$, hence if the CW-complex $X$ is endofinite. Here is a useful criterion:

Theorem C. Let $X$ be a connected $C W$-complex such that the singular homology groups $H_{n}(X ; \mathbb{Z})$ are finite for all $n>0$. Then $X$ is an endofinite $C W$-complex, and therefore its suspension spectrum is also endofinite.

Important examples of CW-complexes with finite homology are the classifying spaces of finite groups.

Corollary. The classifying space BG of a finite group $G$ is endofinite. 
It is a well-known consequence of Carlsson's solution of the Segal conjecture [2] that $B G$ splits stably into indecomposable spectra; see [9] for an alternative proof. We recover this result, and our ideal-theoretic approach reduces the problem of finding such splittings to the category of finite spectra.

The concept of endofiniteness has its origin in representation theory of finite dimensional algebras. Following Crawley-Boevey, a module $X$ over some associative ring $\Lambda$ is endofinite if it has finite length when regarded as a module over its endomorphism ring $\operatorname{End}_{\Lambda}(X)$. Such modules have nice decomposition properties and they play a decisive role for the representation type of a finite dimensional algebra; see [3] for an excellent survey. Comparing the category of modules over some associative ring and the stable homotopy category of spectra, one finds that a number of results about endofinite objects carry over from one setting to the other. The reason for this is the fact that both categories can be embedded in an appropriate way into some abelian functor category. This embedding identifies the endofinite objects with certain injective objects having some nice extra properties. This enables us to use classical results about injective objects in abelian Grothendieck categories. However, the additional triangulated structure of the stable homotopy category led to the discovery of some genuine new phenomena. In fact, the ideal-theoretic approach on which Theorems A and B are based appears to be less natural for the category of modules over a ring.

This paper is organized as follows: In Section 1 we introduce cohomological and cofinite ideals for arbitrary triangulated categories and study their basic properties. For a compactly generated triangulated category $\mathcal{C}$, the restricted Yoneda functor

$$
\mathcal{C} \longrightarrow \operatorname{Mod} \mathcal{C}_{0}, \quad X \mapsto H_{X}=\left.\operatorname{Hom}(-, X)\right|_{\mathcal{C}_{0}}
$$

is an important tool which translates the triangulated structure of $\mathcal{C}$ into the abelian structure of the category $\operatorname{Mod} \mathcal{C}_{0}$ of $\mathcal{C}_{0}$-modules. A detailed treatment of this functor can be found in [14], but we recall in Section 2 the basic results and prove that any cohomological ideal $\mathfrak{I}$ in $\mathcal{C}_{0}$ is the annihilator of some canonical object $E_{\mathfrak{I}}$ in $\mathcal{C}$. The correspondence between endofinite objects and cofinite ideals is the main theme of Section 3, which contains the proofs of Theorem A and Theorem B. In the following two sections we apply the results from the previous ones to the stable homotopy category of spectra. In Section 4 we investigate $\mathfrak{p}$-localizations of endofinite spectra and introduce the endolength of a spectrum $X$, which is a numerical invariant, i.e. a family $\left(\ell_{n}(X)\right)_{n \in \mathbb{Z}}$ of numbers $\ell_{n}(X) \in \mathbb{N} \cup\{\infty\}$ such that $X$ is endofinite if and only if $\ell_{n}(X)$ is finite for all $n \in \mathbb{Z}$. The final Section 5 is devoted to the criterion for a CW-complex to be endofinite, which is formulated in Theorem C.

\section{Cohomological ideals}

In this section we introduce cohomological and cofinite ideals for arbitrary triangulated categories, and study their basic properties. We use the Yoneda functor, which embeds a triangulated category into an abelian category.

1.1. The Yoneda functor. Let $\mathcal{C}$ be any additive category. A $\mathcal{C}$-module is by definition an additive functor $\mathcal{C}^{\mathrm{op}} \rightarrow \mathrm{Ab}$ into the category $\mathrm{Ab}$ of abelian groups, and for $\mathcal{C}$-modules $M$ and $N$ we denote by $\operatorname{Hom}(M, N)$ the class of natural transformations $M \rightarrow N$. A sequence $L \rightarrow M \rightarrow N$ of maps between $\mathcal{C}$-modules is exact if the sequence $L(X) \rightarrow M(X) \rightarrow N(X)$ is exact for all $X$ in $\mathcal{C}$. A $\mathcal{C}$-module $M$ is finitely generated if there exists an exact sequence $\operatorname{Hom}(-, X) \rightarrow M \rightarrow 0$ 
for some $X$ in $\mathcal{C}$, and $M$ is finitely presented if there exists an exact sequence $\operatorname{Hom}(-, X) \rightarrow \operatorname{Hom}(-, Y) \rightarrow M \rightarrow 0$ with $X$ and $Y$ in $\mathcal{C}$. Note that $\operatorname{Hom}(M, N)$ is a set for every finitely generated $\mathcal{C}$-module $M$ by Yoneda's lemma. The finitely presented $\mathcal{C}$-modules form an additive category with cokernels, which we denote by $\bmod \mathcal{C}$. It is well-known (and an easy exercise) that $\bmod \mathcal{C}$ is abelian if and only if every map $Y \rightarrow Z$ in $\mathcal{C}$ has a weak kernel $X \rightarrow Y$, i.e. the sequence $\operatorname{Hom}(-, X) \rightarrow \operatorname{Hom}(-, Y) \rightarrow \operatorname{Hom}(-, Z)$ is exact. In particular, $\bmod \mathcal{C}$ is abelian if $\mathcal{C}$ is triangulated.

Suppose now that $\mathcal{C}$ is a triangulated category [18. Any map $\psi: Y \rightarrow Z$ in $\mathcal{C}$ can be completed to a triangle

$$
X \stackrel{\phi}{\longrightarrow} Y \stackrel{\psi}{\longrightarrow} Z \stackrel{\chi}{\longrightarrow} X[1]
$$

and we call the map $\phi$ a fibre of $\psi$ and $\chi$ a cofibre of $\psi$. Recall that a functor $F: \mathcal{C} \rightarrow \mathcal{A}$ from $\mathcal{C}$ into an abelian category $\mathcal{A}$ is cohomological if for every triangle $(*)$ the sequence $F(X) \rightarrow F(Y) \rightarrow F(Z) \rightarrow F(X[1])$ is exact. Examples of cohomological functors are the representable functors $\operatorname{Hom}(X,-): \mathcal{C} \rightarrow \mathrm{Ab}$ and $\operatorname{Hom}(-, X): \mathcal{C}^{\text {op }} \rightarrow \mathrm{Ab}$ for any $X$ in $\mathcal{C}$. Another important example is the fully faithful Yoneda functor

$$
H: \mathcal{C} \longrightarrow \bmod \mathcal{C}, \quad X \mapsto H_{X}=\operatorname{Hom}(-, X) .
$$

A map $\phi: X \rightarrow Y$ is sent to the natural transformation $H_{\phi}: \operatorname{Hom}(-, X) \rightarrow$ $\operatorname{Hom}(-, Y)$. The Yoneda functor has the following well-known universal property:

Freyd's Lemma. Every additive functor $F: \mathcal{C} \rightarrow \mathcal{A}$ into an abelian category $\mathcal{A}$ extends, up to isomorphism, uniquely to a right exact functor $F^{\prime}: \bmod \mathcal{C} \rightarrow \mathcal{A}$ such that $F=F^{\prime} \circ H$. The functor $F^{\prime}$ is exact if and only if $F$ is a cohomological functor.

Proof. See [6, Theorem 3.1].

In order to study the category $\bmod \mathcal{C}$ it is important to observe that any object $F$ in $\bmod \mathcal{C}$ is of the form $F=\operatorname{Im} H_{\phi}$ for some map $\phi: Y \rightarrow Z$ in $\mathcal{C}$. In fact, if $H_{X} \rightarrow H_{Y} \rightarrow F \rightarrow 0$ is a presentation of $F$, then $F=\operatorname{Im} H_{\phi}$, where $\phi: Y \rightarrow Z$ denotes a cofibre of the map $X \rightarrow Y$. Moreover, the representable functors $H_{X}$ are projective objects in $\bmod \mathcal{C}$ by Yoneda's lemma, and they are also injective since $\mathcal{C}$ is triangulated. Our next aim is a description of the exact sequences in $\bmod \mathcal{C}$. To this end we make the following definition:

Definition 1.1. A sequence $X^{\prime} \stackrel{\phi^{\prime}}{\rightarrow} X \stackrel{\phi}{\rightarrow} Y \stackrel{\phi^{\prime \prime}}{\rightarrow} Y^{\prime \prime}$ of maps in $\mathcal{C}$ is called cohomological exact if $\phi^{\prime}$ is a fibre of the composition $\phi^{\prime \prime} \phi$.

The following two lemmas explain the relevance of the cohomological exact sequences.

Lemma 1.2. Let $X^{\prime} \stackrel{\phi^{\prime}}{\rightarrow} X \stackrel{\phi}{\rightarrow} Y \stackrel{\phi^{\prime \prime}}{\rightarrow} Y^{\prime \prime}$ be a cohomological exact sequence in $\mathcal{C}$. Then the induced sequence

$$
0 \longrightarrow \operatorname{Im} H_{\phi \phi^{\prime}} \longrightarrow \operatorname{Im} H_{\phi} \longrightarrow \operatorname{Im} H_{\phi^{\prime \prime} \phi} \longrightarrow 0
$$

is exact in $\bmod \mathcal{C}$.

Proof. Clearly, the map $\operatorname{Im} H_{\phi} \rightarrow \operatorname{Im} H_{\phi^{\prime \prime} \phi}$ is an epimorphism, and $\operatorname{Im} H_{\phi \phi^{\prime}}$ is the kernel of this map since $\phi^{\prime}$ is a fibre of $\phi^{\prime \prime} \phi$. 
Lemma 1.3. Let $\varepsilon: 0 \rightarrow F^{\prime} \rightarrow F \rightarrow F^{\prime \prime} \rightarrow 0$ be an exact sequence in $\bmod \mathcal{C}$. Then there exists a cohomological exact sequence $X^{\prime} \stackrel{\phi^{\prime}}{\rightarrow} X \stackrel{\phi}{\rightarrow} Y \stackrel{\phi^{\prime \prime}}{\rightarrow} Y^{\prime \prime}$ in $\mathcal{C}$ such that the induced sequence

$$
0 \longrightarrow \operatorname{Im} H_{\phi \phi^{\prime}} \longrightarrow \operatorname{Im} H_{\phi} \longrightarrow \operatorname{Im} H_{\phi^{\prime \prime} \phi} \longrightarrow 0
$$

is isomorphic to $\varepsilon$.

Proof. Let $F=\operatorname{Im} H_{\phi}$ for some map $\phi: X \rightarrow Y$. Taking a monomorphism $F^{\prime \prime} \rightarrow$ $H_{Y^{\prime \prime}}$, the composition $F \rightarrow F^{\prime \prime} \rightarrow H_{Y^{\prime \prime}}$ extends to a map $H_{Y} \rightarrow H_{Y^{\prime \prime}}$ since $H_{Y^{\prime \prime}}$ is injective. By Yoneda's lemma, this map is induced by a map $\phi^{\prime \prime}: Y \rightarrow Y^{\prime \prime}$, and it follows that $F^{\prime \prime}=\operatorname{Im} H_{\phi^{\prime \prime} \phi}$. Taking for $\phi^{\prime}: X^{\prime} \rightarrow X$ a fibre of the composition $\phi^{\prime \prime} \phi$, it follows from the preceding lemma that the induced sequence

$$
0 \longrightarrow \operatorname{Im} H_{\phi \phi^{\prime}} \longrightarrow \operatorname{Im} H_{\phi} \longrightarrow \operatorname{Im} H_{\phi^{\prime \prime} \phi} \longrightarrow 0
$$

is isomorphic to $\varepsilon$.

1.2. Cohomological ideals. Let $\mathcal{C}$ be a triangulated category. An ideal $\mathfrak{I}$ in $\mathcal{C}$ consists of subgroups $\mathfrak{I}(X, Y)$ in $\operatorname{Hom}(X, Y)$ for every pair of objects $X, Y$ in $\mathcal{C}$ such that for all $\phi$ in $\mathfrak{I}(X, Y)$ and all maps $\alpha: X^{\prime} \rightarrow X$ and $\beta: Y \rightarrow Y^{\prime}$ in $\mathcal{C}$ the composition $\beta \phi \alpha$ belongs to $\Im\left(X^{\prime}, Y^{\prime}\right)$. Given an additive functor $F: \mathcal{C} \rightarrow \mathcal{D}$, the ideal of maps $\phi$ in $\mathcal{C}$ satisfying $F(\phi)=0$ is called the annihilator of $F$ and is denoted by Ann $F$. Note that any ideal $\mathfrak{I}$ in $\mathcal{C}$ is of the form $\mathfrak{I}=\operatorname{Ann} F$ for some functor $F: \mathcal{C} \rightarrow \mathcal{D}$, since we can take for $F$ the projection $\mathcal{C} \rightarrow \mathcal{C} / \mathfrak{I}$ onto the additive quotient category $\mathcal{C} / \mathfrak{I}$.

Definition 1.4. An ideal in $\mathcal{C}$ is said to be cohomological if it is the annihilator of some cohomological functor $\mathcal{C} \rightarrow \mathcal{A}$.

For example, if $F: \mathcal{C} \rightarrow \mathcal{D}$ is an exact functor between triangulated categories, then $\operatorname{Ann} F$ is a cohomological ideal since $\operatorname{Ann} F=\operatorname{Ann}(H \circ F)$, where $H: \mathcal{D} \rightarrow$ $\bmod \mathcal{D}$ denotes the Yoneda functor, which is faithful.

Recall that a full subcategory $\mathcal{S}$ of an abelian category $\mathcal{A}$ is a Serre subcategory provided that for every exact sequence $0 \rightarrow X^{\prime} \rightarrow X \rightarrow X^{\prime \prime} \rightarrow 0$ in $\mathcal{A}$ the object $X$ belongs to $\mathcal{S}$ if and only if $X^{\prime}$ and $X^{\prime \prime}$ belong to $\mathcal{S}$. The corresponding quotient category $\mathcal{A} / \mathcal{S}$ is constructed as follows $[7$ : The objects of $\mathcal{A} / \mathcal{S}$ are those of $\mathcal{A}$, and

$$
\operatorname{Hom}_{\mathcal{A} / \mathcal{S}}(X, Y)=\lim _{\operatorname{Hom}} \operatorname{Hom}_{\mathcal{A}}\left(X^{\prime}, Y / Y^{\prime}\right)
$$

with $X^{\prime} \subseteq X, Y^{\prime} \subseteq Y$ and $X / X^{\prime}, Y^{\prime} \in \mathcal{S}$. Note that the maps in $\mathcal{A} / \mathcal{S}$ form a set if one assumes that the subobjects of any fixed object in $\mathcal{A}$ form a set. Again the category $\mathcal{A} / \mathcal{S}$ is abelian, and there is canonically defined the quotient functor $Q: \mathcal{A} \rightarrow \mathcal{A} / \mathcal{S}$ such that $Q(X)=X$; it is exact with $\operatorname{Ker} Q=\mathcal{S}$. Here the kernel Ker $F$ of a functor $F: \mathcal{A} \rightarrow \mathcal{B}$ is, by definition, the full subcategory of all objects $X$ in $\mathcal{A}$ such that $F(X) \cong 0$. Now suppose that $F: \mathcal{A} \rightarrow \mathcal{B}$ is an exact functor. Then Ker $F$ contains $\mathcal{S}$ if and only if $F$ induces a (unique and exact) functor $G: \mathcal{A} / \mathcal{S} \rightarrow \mathcal{B}$ such that $F=G \circ Q$. Moreover, $G$ is faithful if and only if $\mathcal{S}=\operatorname{Ker} F$.

Proposition 1.5. The assignments

$$
\mathfrak{I} \mapsto \mathcal{S}_{\mathfrak{I}}=\left\{\operatorname{Im} H_{\phi} \mid \phi \in \mathfrak{I}\right\} \quad \text { and } \quad \mathcal{S} \mapsto \mathfrak{I}_{\mathcal{S}}=\left\{\phi \in \mathcal{C} \mid \operatorname{Im} H_{\phi} \in \mathcal{S}\right\}
$$

induce mutually inverse bijections between the class of cohomological ideals in $\mathcal{C}$ and the class of Serre subcategories in $\bmod \mathcal{C}$. 
Proof. Let $\mathcal{A}=\bmod \mathcal{C}$. Suppose that $\mathfrak{I}=\operatorname{Ann} F$ for some cohomological functor $F: \mathcal{C} \rightarrow \mathcal{A}^{\prime}$ and denote by $F^{\prime}: \mathcal{A} \rightarrow \mathcal{A}^{\prime}$ the unique exact functor extending $F$, which exists by Freyd's lemma. Clearly, Ker $F^{\prime}=\mathcal{S}_{\mathfrak{I}}$, and this is a Serre subcategory of $\mathcal{A}$ since $F^{\prime}$ is exact. Conversely, if $\mathcal{S}$ is a Serre subcategory of $\mathcal{A}$, then $\mathfrak{I}_{\mathcal{S}}=\operatorname{Ann} F$, where $F: \mathcal{C} \rightarrow \mathcal{A} / \mathcal{S}$ denotes the composition of the Yoneda functor $\mathcal{C} \rightarrow \mathcal{A}$ with the quotient functor $\mathcal{A} \rightarrow \mathcal{A} / \mathcal{S}$. It is easily checked that these constructions are mutually inverse.

As a consequence of this proposition, we obtain an internal characterization of cohomological ideals in terms of maps in $\mathcal{C}$.

Corollary 1.6. An ideal $\mathfrak{I}$ in $\mathcal{C}$ is cohomological if and only if for every cohomological exact sequence in $\mathcal{C}$

$$
X^{\prime} \stackrel{\phi^{\prime}}{\longrightarrow} X \stackrel{\phi}{\longrightarrow} Y \stackrel{\phi^{\prime \prime}}{\longrightarrow} Y^{\prime \prime}
$$

the map $\phi$ belongs to $\mathfrak{I}$ provided that $\phi \phi^{\prime}$ and $\phi^{\prime \prime} \phi$ are in $\mathfrak{I}$.

Proof. Combine the description of exact sequences in $\bmod \mathcal{C}$ given in Lemma 1.2 and Lemma 1.3 with Proposition 1.5

1.3. Cofinite ideals. Let $\mathcal{C}$ be a triangulated category and let $\mathcal{A}=\bmod \mathcal{C}$. Recall that an abelian category is a length category if every object has finite composition length.

Definition 1.7. Let $\mathfrak{I}$ be a cohomological ideal in $\mathcal{C}$, and let $\mathcal{S}_{\mathfrak{I}}$ be the corresponding Serre subcategory of $\mathcal{A}$. Then $\mathfrak{I}$ is called cofinite if the quotient category $\mathcal{A} / \mathcal{S}_{\mathfrak{I}}$ is a length category.

In order to give an internal characterization of cofinite ideals in $\mathcal{C}$ we need another definition.

Definition 1.8. Let $\mathfrak{I}$ be a cohomological ideal in $\mathcal{C}$.

(1) An $\mathfrak{I}$-sequence is a sequence of maps $X \stackrel{\phi_{0}}{\longrightarrow} X_{0} \stackrel{\phi_{1}}{\longrightarrow} \ldots \stackrel{\phi_{n}}{\longrightarrow} X_{n}$ in $\mathcal{C}$ such that for every $i \in\{1, \ldots, n\}$ the composition of $\phi_{i-1} \ldots \phi_{0}$ with a fibre of $\phi_{i} \ldots \phi_{0}$ does not belong to $\mathfrak{I}$.

(2) The $\mathfrak{I}$-length of an object $X$ in $\mathcal{C}$ is

$$
\ell_{\mathfrak{I}}(X)=\sup \left\{n \in \mathbb{N} \mid \text { there exists an } \mathfrak{I} \text {-sequence } X \stackrel{\phi_{0}}{\longrightarrow} X_{0} \stackrel{\phi_{1}}{\longrightarrow} \ldots \stackrel{\phi_{n}}{\rightarrow} X_{n}\right\} \text {. }
$$

We are now in a position to state a characterization of cofinite ideals in $\mathcal{C}$.

Proposition 1.9. A cohomological ideal $\mathfrak{I}$ in $\mathcal{C}$ is cofinite if and only if the $\mathfrak{I}$-length of every object in $\mathcal{C}$ is finite.

The proof of this proposition requires two lemmas.

Lemma 1.10. Let $0=F_{0} \subseteq F_{1} \subseteq \ldots \subseteq F_{n}=H_{X}$ be a chain of subobjects in $\mathcal{A}$.

(1) There exists a sequence $X \stackrel{\phi_{0}}{\rightarrow} X_{0} \stackrel{\phi_{1}}{\rightarrow} \ldots \stackrel{\phi_{n}}{\rightarrow} X_{n}$ of maps in $\mathcal{C}$ such that $F_{i}=\operatorname{Ker} H_{\phi_{i} \ldots \phi_{0}}$ for all $i$.

(2) Denote for every $i$ by $\psi_{i}$ a fibre of $\phi_{i} \ldots \phi_{0}$. Then $F_{i} / F_{i-1} \cong \operatorname{Im} H_{\phi_{i-1} \ldots \phi_{0} \psi_{i}}$ for all $i$. 
Proof. (1) Put $X_{0}=X$ and $\phi_{0}=\mathrm{id}_{X}$. Choose for every $i>0$ a copresentation $0 \rightarrow F_{i} \rightarrow H_{X} \rightarrow H_{X_{i}}$. The inclusion $F_{i-1} \rightarrow F_{i}$ induces a commutative diagram

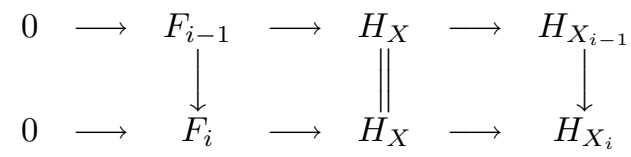

and the map $H_{X_{i-1}} \rightarrow H_{X_{i}}$ is induced by a map $\phi_{i}: X_{i-1} \rightarrow X_{i}$. This gives the desired sequence $X \stackrel{\phi_{0}}{\rightarrow} X_{0} \stackrel{\phi_{1}}{\rightarrow} \ldots \stackrel{\phi_{n}}{\rightarrow} X_{n}$.

(2) An application of the snake lemma shows that $F_{i} / F_{i-1}$ is isomorphic to the kernel of the map $\operatorname{Im} H_{\phi_{i-1} \ldots \phi_{0}} \rightarrow \operatorname{Im} H_{\phi_{i} \ldots \phi_{0}}$, which is $\operatorname{Im} H_{\phi_{i-1} \ldots \phi_{0} \psi_{i}}$ by Lemma 1.2

Lemma 1.11. Let $\mathfrak{I}$ be a cohomological ideal and $X \in \mathcal{C}$. Then $\ell_{\mathfrak{I}}(X)$ is the length of $H_{X}$ in $\mathcal{A} / \mathcal{S}_{\mathfrak{J}}$.

Proof. Let $Q: \mathcal{A} \rightarrow \mathcal{A} / \mathcal{S}_{\mathfrak{I}}$ be the quotient functor. Any chain of subobjects of $Q\left(H_{X}\right)$ is the image of a chain $0=F_{0} \subseteq F_{1} \subseteq \ldots \subseteq F_{n}=H_{X}$ in $\mathcal{A}$, and we denote by $X \stackrel{\phi_{0}}{\longrightarrow} X_{0} \stackrel{\phi_{1}}{\longrightarrow} \ldots \stackrel{\phi_{n}}{\longrightarrow} X_{n}$ the sequence in $\mathcal{C}$ with $F_{i}=$ Ker $H_{\phi_{i} \ldots \phi_{0}}$, which exists by Lemma 1.10, By construction, $Q\left(F_{i}\right) / Q\left(F_{i-1}\right) \neq 0$ if and only if $F_{i} / F_{i-1} \notin \mathcal{S}_{\mathfrak{J}}$. Furthermore, $F_{i} / F_{i-1} \notin \mathcal{S}_{\mathfrak{I}}$ if and only if the composition of $\phi_{i-1} \ldots \phi_{0}$ with a fibre of $\phi_{i} \ldots \phi_{0}$ does not belong to $\mathfrak{I}$, by Lemma 1.10. Thus $\ell_{\mathfrak{I}}(X)$ is the length of $Q\left(H_{X}\right)$.

Proof of Proposition 1.9. Any object in $\mathcal{A} / \mathcal{S}_{\mathfrak{I}}$ is the quotient of $H_{X}$ for some $X$ in $\mathcal{C}$. Therefore $\mathcal{A} / \mathcal{S}_{\mathfrak{I}}$ is a length category if and only if $\ell_{\mathfrak{I}}(X)$ is finite for all $X$, by Lemma 1.11

Now suppose that $\mathfrak{I}$ is a cofinite cohomological ideal in $\mathcal{C}$, and denote by $Q: \mathcal{A} \rightarrow$ $\mathcal{A} / \mathcal{S}_{\mathfrak{I}}$ the corresponding quotient functor. Then the assignment

$$
X \mapsto \mathcal{S}_{X}=\{A \in \mathcal{A} \mid X \text { does not occur as a composition factor of } Q(A)\}
$$

induces a bijection between the isomorphism classes of simple objects in $\mathcal{A} / \mathcal{S}_{\mathfrak{I}}$ and the maximal Serre subcategories $\mathcal{S} \neq \mathcal{A}$ of $\mathcal{A}$ containing $\mathcal{S}_{\mathfrak{I}}$. We include the following consequence for later reference.

Lemma 1.12. Let $\mathfrak{I}$ be a cofinite cohomological ideal in $\mathcal{C}$. The map $X \mapsto \mathfrak{I}_{\mathcal{S}_{X}}$ induces a bijection between the isomorphism classes of simple objects in $\mathcal{A} / \mathcal{S}_{\mathfrak{I}}$ and the maximal cohomological ideals $\mathfrak{J} \neq \mathcal{C}$ in $\mathcal{C}$ containing $\mathfrak{I}$.

Proof. Apply the inclusion-preserving bijection from Proposition 1.5 between Serre subcategories of $\mathcal{A}$ and cohomological ideals in $\mathcal{C}$.

\section{ANNIHILATOR IDEALS}

Let $\mathcal{C}$ be a compactly generated triangulated category [17. More precisely, $\mathcal{C}$ is a triangulated category and has arbitrary coproducts. An object $X$ in $\mathcal{C}$ is called compact if for every family $\left(Y_{i}\right)_{i \in I}$ in $\mathcal{C}$ the canonical map $\coprod_{i} \operatorname{Hom}\left(X, Y_{i}\right) \rightarrow$ $\operatorname{Hom}\left(X, \coprod_{i} Y_{i}\right)$ is an isomorphism. We denote by $\mathcal{C}_{0}$ the full subcategory of compact objects in $\mathcal{C}$, and observe that $\mathcal{C}_{0}$ is a triangulated subcategory of $\mathcal{C}$. For $\mathcal{C}$ compactly generated the isomorphism classes of objects in $\mathcal{C}_{0}$ need to form a set, and $\operatorname{Hom}(C, X)=0$ for all $C$ in $\mathcal{C}_{0}$ implies $X=0$ for every object $X$ in $\mathcal{C}$. 
2.1. The restricted Yoneda functor. We denote by $\operatorname{Mod} \mathcal{C}_{0}$ the category of $\mathcal{C}_{0^{-}}$ modules, and our main tool for studying the category $\mathcal{C}$ is the restricted Yoneda functor

$$
\mathcal{C} \longrightarrow \operatorname{Mod} \mathcal{C}_{0}, \quad X \mapsto H_{X}=\left.\operatorname{Hom}(-, X)\right|_{\mathcal{C}_{0}} .
$$

We need to recall the concept of purity for a compactly generated triangulated category which has been introduced in [14]. A map $X \rightarrow Y$ in $\mathcal{C}$ is a pure monomorphism if the induced map $\operatorname{Hom}(C, X) \rightarrow \operatorname{Hom}(C, Y)$ is a monomorphism for all $C$ in $\mathcal{C}_{0}$. An object $X$ in $\mathcal{C}$ is pure-injective if every pure monomorphism $X \rightarrow Y$ is a split monomorphism. The following lemma summarizes some essential properties of the restricted Yoneda functor.

Lemma 2.1. The restricted Yoneda functor $\mathcal{C} \rightarrow \operatorname{Mod} \mathcal{C}_{0}$ identifies the pure-injectives in $\mathcal{C}$ with the injectives in $\operatorname{Mod} \mathcal{C}_{0}$. Moreover, an object $X$ in $\mathcal{C}$ is pure-injective if and only if the map $\operatorname{Hom}(Y, X) \rightarrow \operatorname{Hom}\left(H_{Y}, H_{X}\right), \phi \mapsto H_{\phi}$, is bijective for every $Y$ in $\mathcal{C}$. A map $\phi$ in $\mathcal{C}$ is a pure monomorphism if and only if $H_{\phi}$ is a monomorphism in $\operatorname{Mod} \mathcal{C}_{0}$.

Proof. See Corollary 1.9 and Lemma 1.7 in [14].

We denote by $\mathrm{Sp} \mathcal{C}$ the set of isomorphism classes of indecomposable pure-injectives in $\mathcal{C}$, and by $\operatorname{Spec} \mathcal{C}_{0}$ the set of isomorphism classes of indecomposable injectives in $\operatorname{Mod} \mathcal{C}_{0}$.

2.2. The annihilator. We define the annihilator of an object in $\mathcal{C}$ as follows:

Definition 2.2. The annihilator $\operatorname{Ann} X$ of an object $X$ in $\mathcal{C}$ is the annihilator of the functor $\left.\operatorname{Hom}(-, X)\right|_{\mathcal{C}_{0}}$.

Note that $\operatorname{Ann} X$ is a cohomological ideal in $\mathcal{C}_{0}$, since $\left.\operatorname{Hom}(-, X)\right|_{\mathcal{C}_{0}}$ is a cohomological functor. Given a cohomological ideal $\mathfrak{I}$ in $\mathcal{C}_{0}$, we denote by $E_{\mathfrak{I}}$ the product of all $X$ in $\operatorname{Sp} \mathcal{C}$ with $\mathfrak{I} \subseteq \operatorname{Ann} X$. The following result shows that the indecomposable pure-injectives in $\mathcal{C}$ control the cohomological ideals in $\mathcal{C}_{0}$.

Theorem 2.3. Let $\mathfrak{I}$ be a cohomological ideal in $\mathcal{C}_{0}$ and $X$ be an object in $\mathcal{C}$.

(1) $\mathfrak{I}=\operatorname{Ann} E_{\mathfrak{I}}$.

(2) $\mathfrak{I} \subseteq$ Ann $X$ if and only if there exists a pure monomorphism $X \rightarrow \prod_{I} E_{\mathfrak{I}}$ for some set $I$.

The theorem is a consequence of a result about the category of $\mathcal{C}_{0}$-modules which we take from [12].

Lemma 2.4. Let $\mathcal{S}$ be a Serre subcategory of $\bmod \mathcal{C}_{0}$ and let $E_{\mathcal{S}}$ be the product of all $Y \in \operatorname{Spec} \mathcal{C}_{0}$ with $\operatorname{Hom}(\mathcal{S}, Y)=0$. Also, let $X$ be an object in $\operatorname{Mod} \mathcal{C}_{0}$.

(1) If $X \in \bmod \mathcal{C}_{0}$, then $X \in \mathcal{S}$ if and only if $\operatorname{Hom}\left(X, E_{\mathcal{S}}\right)=0$.

(2) $\operatorname{Hom}(\mathcal{S}, X)=0$ if and only if there exists a monomorphism $X \rightarrow \prod_{I} E_{\mathcal{S}}$ for some set $I$.

Proof. See [12, Proposition 3.2].

Proof of Theorem [2.3. Let $\mathcal{S}=\mathcal{S}_{\mathfrak{I}}$ be the Serre subcategory of $\bmod \mathcal{C}_{0}$ corresponding to $\mathfrak{I}$. Given a map $\phi$ in $\mathcal{C}_{0}$ and an object $X$ in $\mathcal{C}$, we have $\phi \in \operatorname{Ann} X$ if and only if $\operatorname{Hom}\left(\operatorname{Im} H_{\phi}, H_{X}\right)=0$; in particular, $\mathfrak{I} \subseteq \operatorname{Ann} X$ if and only if $\operatorname{Hom}\left(\mathcal{S}, H_{X}\right)=0$. Therefore the restricted Yoneda functor $\mathcal{C} \rightarrow \operatorname{Mod} \mathcal{C}_{0}$ identifies $E_{\mathfrak{I}}$ with $E_{\mathcal{S}}$ and 
induces a bijection $\operatorname{Hom}\left(X, E_{\mathfrak{I}}\right) \rightarrow \operatorname{Hom}\left(H_{X}, E_{\mathcal{S}}\right)$ for every $X$ in $\mathcal{C}$ by Lemma 2.1 The assertion of the theorem now follows from these observations and the preceding lemma.

\section{ENDOFINITENESS}

Let $\mathcal{C}$ be a compactly generated triangulated category. The notion of an endofinite object has been introduced by Crawley-Boevey for so-called locally finitely presented additive categories [4]. An analogue of this concept for compactly generated triangulated categories has been studied in [15]. Let us recall this definition.

Definition 3.1. An object $X$ in $\mathcal{C}$ is called endofinite if for every compact object $C$ in $\mathcal{C}$ the $\operatorname{End}(X)$-module $\operatorname{Hom}(C, X)$ has finite composition length.

3.1. A characterization. We have the following characterization of endofinite objects in terms of cohomological ideals.

Theorem 3.2. An object $X$ in $\mathcal{C}$ is endofinite if and only if the annihilator of $X$ is a cofinite ideal in $\mathcal{C}_{0}$.

Proof. Let $\Lambda_{X}=\operatorname{End}(X)$ and $\Gamma_{X}=\operatorname{End}\left(H_{X}\right)$. We define $\mathcal{A}=\bmod \mathcal{C}_{0}$ and observe that the map $\operatorname{Hom}(-, C) \mapsto \operatorname{Hom}(C,-)$ induces an equivalence $\mathcal{A}^{\mathrm{op}} \rightarrow \bmod \left(\mathcal{C}_{0}^{\mathrm{op}}\right)$. Thus we can view the $\mathcal{C}_{0}$-module $H_{X}: \mathcal{C}_{0}^{\text {op }} \rightarrow \mathrm{Ab}$ as an exact functor $F_{X}: \mathcal{A}^{\mathrm{op}} \rightarrow \mathrm{Ab}$ by Freyd's lemma. In fact, $F_{X}$ is an exact functor $\mathcal{A}^{\mathrm{op}} \rightarrow \operatorname{Mod} \Gamma_{X}$ in the obvious way, and the Serre subcategory $\mathcal{S}=\mathcal{S}_{\operatorname{Ann} X}$ of $\mathcal{A}$ corresponding to Ann $X$ is the kernel of $F_{X}$. In [13, Theorem 10.2] it is shown that $F_{X}(A)$ is a finite length $\Gamma_{X^{-}}$ module for every $A$ in $\mathcal{A}$ if and only if $\mathcal{A} / \mathcal{S}$ is a length category. Suppose now that $X$ is endofinite. Then $F_{X}(A)$ is for every $A$ in $\mathcal{A}$ a finite length $\Lambda_{X}$-module, and therefore also a finite length $\Gamma_{X}$-module since the $\Lambda_{X}$-action is induced by the canonical map $\Lambda_{X} \rightarrow \Gamma_{X}$. Thus $\mathcal{A} / \mathcal{S}$ is a length category and Ann $X$ is cofinite. Conversely, if Ann $X$ is cofinite, then $F_{X}(A)$ is a finite length $\Gamma_{X}$-module for every $A$ in $\mathcal{A}$. It follows from [13, Theorem 9.6] and the characterization of pure-injectives in [14, Theorem 1.8] that $X$ is pure-injective. Thus the canonical map $\Lambda_{X} \rightarrow \Gamma_{X}$ is an isomorphism by Lemma 2.1, and therefore $F_{X}(A)$ is of finite length over $\Lambda_{X}$ for all $A$. We conclude that $X$ is endofinite, and this finishes the proof.

3.2. Decomposing endofinite objects. Endofinite objects have nice decomposition properties. We recall the basic result, established in [15].

Proposition 3.3. An endofinite object $X$ is pure-injective and has, up to isomorphism, a unique decomposition $X \cong \coprod_{i \in I} X_{i}$ into indecomposable objects with local endomorphism ring.

Proof. See [15, Theorem 1.2].

The indecomposable endofinite objects can be described in terms of certain cohomological ideals. In order to state this result we denote by $\operatorname{Max} \mathcal{C}_{0}$ the set of maximal elements among the cofinite cohomological ideals $\mathfrak{I} \neq \mathcal{C}_{0}$ in $\mathcal{C}_{0}$.

Theorem 3.4. The assignments $\mathfrak{I} \mapsto E_{\mathfrak{I}}$ and $X \mapsto \operatorname{Ann} X$ induce mutually inverse bijections between $\operatorname{Max} \mathcal{C}_{0}$ and the set of isomorphism classes of indecomposable endofinite objects in $\mathcal{C}$. 
Proof. Suppose that $\mathfrak{I}$ is a cofinite cohomological ideal in $\mathcal{C}_{0}$ and let $\mathcal{S}=\mathcal{S}_{\mathfrak{I}}$ be the corresponding Serre subcategory of $\mathcal{A}=\bmod \mathcal{C}_{0}$. Then $\mathcal{A} / \mathcal{S}$ is a length category. We denote by $\mathcal{T}$ the localizing subcategory of $\operatorname{Mod} \mathcal{C}_{0}$ which is generated by $\mathcal{S}$, i.e. $\mathcal{T}$ is the smallest Serre subcategory of $\operatorname{Mod} \mathcal{C}_{0}$ containing $\mathcal{S}$ which is closed under taking coproducts. The quotient category $\operatorname{Mod} \mathcal{C}_{0} / \mathcal{T}$ is locally finite in the sense of [7, II.4], since the quotient functor $Q: \operatorname{Mod} \mathcal{C}_{0} \rightarrow \operatorname{Mod} \mathcal{C}_{0} / \mathcal{T}$ sends $\mathcal{A}$ to a full subcategory of $\operatorname{Mod} \mathcal{C}_{0} / \mathcal{T}$ which is equivalent to $\mathcal{A} / \mathcal{S}$ and generates $\operatorname{Mod} \mathcal{C}_{0} / \mathcal{T}$; see [12, Theorem 2.6] for details. In fact, $\mathcal{A} / \mathcal{S}$ is equivalent to the full subcategory of finite length objects in $\operatorname{Mod} \mathcal{C}_{0} / \mathcal{T}$. The quotient functor $Q$ identifies the indecomposable injective objects $X$ in $\operatorname{Mod} \mathcal{C}_{0}$ satisfying $\operatorname{Hom}(\mathcal{S}, X)=0$ with the indecomposable injective objects in $\operatorname{Mod} \mathcal{C}_{0} / \mathcal{T}$ [7, III.3]. Therefore the indecomposable pure-injective objects $X$ in $\mathcal{C}$ satisfying $\mathfrak{I} \subseteq$ Ann $X$ are identified via $X \mapsto Q\left(H_{X}\right)$ with the indecomposable injective objects in $\operatorname{Mod} \mathcal{C}_{0} / \mathcal{T}$ by Lemma 2.1 Every indecomposable injective object in a locally finite category is the injective envelope of a simple object [7, IV.1]. Therefore $\operatorname{card}\{X \in \operatorname{Sp} \mathcal{C} \mid \mathfrak{I} \subseteq \operatorname{Ann} X\}=1$ if and only if $\mathcal{A} / \mathcal{S}$ has a unique simple object. The cofinite cohomological ideal $\mathfrak{I}$ is maximal if and only if $\mathcal{A} / \mathcal{S}$ has a unique simple object by Lemma 1.12 and therefore $E_{\mathfrak{I}}$ is indecomposable if and only if $\mathfrak{I}$ is a maximal cofinite cohomological ideal in $\mathcal{C}_{0}$. Moreover, $E_{\mathfrak{I}}$ is endofinite by Theorem 3.2 since $\mathfrak{I}=A n n E_{\mathfrak{I}}$, which holds by Theorem 2.3.

Now suppose that $X \in \mathrm{Sp} \mathcal{C}$ is endofinite, and let $\mathfrak{I}=\operatorname{Ann} X$, which is cofinite by Theorem 3.2. Let $S$ be a simple object in $\mathcal{A} / \mathcal{S}_{\mathfrak{I}}$, which we view as a simple object in $\operatorname{Mod} \mathcal{C}_{0} / \mathcal{T}$. By construction, $\operatorname{Hom}\left(S, Q\left(H_{X}\right)\right) \neq 0$, and therefore $Q\left(H_{X}\right)$ is isomorphic to an injective envelope of $S$. It follows that $S$ is the unique simple object in $\mathcal{A} / \mathcal{S}_{\mathfrak{I}}$. Thus $\mathfrak{I}$ is a maximal cofinite cohomological ideal in $\mathcal{C}_{0}$; in particular $X=E_{\mathfrak{I}}$. This finishes the proof.

We discuss now a number of consequences of Theorem 3.2 and Theorem 3.4. The following lemma will be useful.

Lemma 3.5. Let $\left(X_{i}\right)_{i \in I}$ be a family of objects in $\mathcal{C}$. Then

$$
\operatorname{Ann} \coprod_{i \in I} X_{i}=\bigcap_{i \in I} \operatorname{Ann} X_{i}=\operatorname{Ann} \prod_{i \in I} X_{i} \text {. }
$$

Proof. Use the isomorphisms $\coprod_{i} \operatorname{Hom}\left(C, X_{i}\right) \cong \operatorname{Hom}\left(C, \coprod_{i} X_{i}\right)$ and $\operatorname{Hom}\left(C, \prod_{i} X_{i}\right)$ $\cong \prod_{i} \operatorname{Hom}\left(C, X_{i}\right)$ for $C$ in $\mathcal{C}_{0}$.

Corollary 3.6. Let $X$ be an endofinite object in $\mathcal{C}$ with $\mathfrak{I}=\operatorname{Ann} X$. Then

$$
\mathfrak{I}=\bigcap_{\mathfrak{I} \subseteq \mathfrak{J} \in \operatorname{Max} \mathcal{C}_{0}} \mathfrak{J}
$$

and $X$ has a unique decomposition

$$
X \cong \coprod_{\mathfrak{I} \subseteq \mathfrak{J} \in \operatorname{Max} \mathcal{C}_{0}} X_{\mathfrak{J}}
$$

such that each $X_{\mathfrak{J}}$ is a non-empty coproduct of copies of $E_{\mathfrak{J}}$.

Proof. Let $X \cong \coprod_{i \in I} X_{i}$ be the decomposition into indecomposable objects, which exists by Proposition 3.3 The annihilator Ann $X_{i}$ contains Ann $X$ and is therefore 
cofinite by Theorem 3.2, Thus $X_{i}$ is endofinite for all $i$. Applying Theorem 3.4 and Lemma 3.5, we obtain

$$
\mathfrak{I}=\operatorname{Ann} X=\bigcap_{i \in I} \operatorname{Ann} X_{i}=\bigcap_{\mathfrak{I} \subseteq \mathfrak{J} \in \operatorname{Max} \mathcal{C}_{0}} \mathfrak{J} .
$$

Now define, for every $\mathfrak{J} \in \operatorname{Max} \mathcal{C}_{0}$ containing $\mathfrak{I}$,

$$
X_{\mathfrak{J}}=\coprod_{\operatorname{Ann} X_{i}=\mathfrak{J}} X_{i}
$$

Clearly, this gives the desired decomposition

$$
X \cong \coprod_{\mathfrak{I} \subseteq \mathfrak{J} \in \operatorname{Max} \mathcal{C}_{0}} X_{\mathfrak{J}}
$$

It remains to show that $X_{\mathfrak{J}} \neq 0$ for every $\mathfrak{J} \in \operatorname{Max} \mathcal{C}_{0}$ containing $\mathfrak{I}$. We apply the ideas from the proof of Theorem 3.4 and keep the same notation. We fix $\mathfrak{J}$. The ideal $\mathfrak{J}$ corresponds to a simple object $S$ in $\mathcal{A} / \mathcal{S}_{\mathfrak{I}}$ by Lemma 1.12 which we view as a simple object in $\operatorname{Mod} \mathcal{C}_{0} / \mathcal{T}$. The injective envelope of $S$ is $Q\left(H_{E_{\mathfrak{J}}}\right)$, which is isomorphic to a direct factor of $Q\left(H_{X}\right)$ since $\operatorname{Hom}\left(S, Q\left(H_{X}\right)\right) \neq 0$. It follows that $E_{\mathfrak{J}}$ is isomorphic to a direct factor of $X$, $\operatorname{since} \operatorname{Hom}\left(E_{\mathfrak{J}}, X\right) \cong \operatorname{Hom}\left(Q\left(H_{E_{\mathfrak{J}}}\right), Q\left(H_{X}\right)\right)$. The uniqueness of the decomposition $X \cong \coprod_{i \in I} X_{i}$ implies that $X_{i} \cong E_{\mathfrak{J}}$ for some $i \in I$, and therefore $X_{\mathfrak{J}} \neq 0$. This completes the proof.

Corollary 3.7. Let $X$ and $Y$ be objects in $\mathcal{C}$, and suppose that $X$ is endofinite. Then Ann $X \subseteq$ Ann $Y$ if and only if $Y$ is isomorphic to a direct factor of a (co)product of copies of $X$.

Proof. If $Y$ is isomorphic to a direct factor of a (co)product of copies of $X$, then Ann $X \subseteq$ Ann $Y$ by Lemma 3.5. To prove the converse, suppose that Ann $X \subseteq$ Ann $Y$. Observe that $Y$ is endofinite by Theorem 3.2, since Ann $Y$ is cofinite. Let $X \cong \coprod_{i \in I} X_{i}$ and $Y \cong \coprod_{j \in J} Y_{j}$ be the decompositions into indecomposables which exist by Proposition 3.3. It follows from Corollary 3.6 that every factor $Y_{j}$ is isomorphic to $X_{i}$ for some $i \in I$, since $Y_{j} \cong E_{\mathfrak{J}}$ for some $\mathfrak{J} \in \operatorname{Max} \mathcal{C}_{0}$ containing Ann $Y$ and therefore containing Ann $X$. The family of split monomorphisms $Y_{j} \rightarrow X$ induces a split monomorphism $Y \rightarrow \coprod_{J} X$, and the composition with the canonical map $\coprod_{J} X \rightarrow \prod_{J} X$ is again a split monomorphism since it is a pure monomorphism and $Y$ is pure-injective by Proposition 3.3 We conclude that $Y$ is isomorphic to a direct factor of a (co)product of copies of $X$.

Corollary 3.8. An indecomposable object $X$ is endofinite if and only if every product of copies of $X$ is a coproduct of copies of $X$.

Proof. Suppose first that $X$ is indecomposable and endofinite. Any product $\prod_{I} X$ is again endofinite by Theorem 3.2. since Ann $\prod_{I} X=\operatorname{Ann} X$ by Lemma 3.5. The decomposition of $\prod_{I} X$ as a coproduct of copies of $X$ then follows directly from Corollary 3.6 Now suppose that $X$ is any indecomposable object such that every product of copies of $X$ is a coproduct of copies of $X$. We consider the corresponding $\mathcal{C}_{0}$-module $M=H_{X}$, and it is clear that every product of copies of $M$ is a coproduct of copies of $M$. It follows from [8, Théorème] that $M$ is a pure-injective $\mathcal{C}_{0}$-module. In fact, $M$ is injective since $M$ is fp-injective by [14, Lemma 1.6], and therefore every monomorphism $M \rightarrow N$ in $\operatorname{Mod} \mathcal{C}_{0}$ is a pure monomorphism, hence a split monomorphism. It follows from Lemma 2.1 that $X$ is a pure-injective object in $\mathcal{C}$. Viewing $X$ as an exact functor $F_{X}: \mathcal{A}^{\mathrm{op}} \rightarrow \mathrm{Ab}$ for $\mathcal{A}=\bmod \mathcal{C}_{0}$ as in the proof 
of Theorem 3.2, it follows from the characterization of endofinite objects in [13. Corollary 10.5] that $X$ is also an endofinite object in $\mathcal{C}$.

\section{ENDOFinite SPECTRA}

In the following we apply the results of the previous sections to the stable homotopy category $\mathcal{C}$ in the sense of 1 . The objects are $\mathrm{CW}$-spectra, and the morphisms $X \rightarrow Y$ between two spectra $X$ and $Y$ are denoted by $\{X, Y\}$. We identify each $\mathrm{CW}$-complex with its suspension spectrum. The stable homotopy category is a triangulated category where the suspension $X \mapsto \Sigma X=X \wedge S^{1}$ acts as a translation functor. In particular, $S^{n}=\Sigma^{n}\left(S^{0}\right)$ is defined for all $n \in \mathbb{Z}$, and for a spectrum $X$ we denote by $\pi_{n}^{s}(X)$ the $n$-th stable homotopy group $\left\{S^{n}, X\right\}$. Coproducts are given by one-point unions called wedge, and arbitrary coproducts do exist in $\mathcal{C}$. Recall that a spectrum $X$ is finite if for some $n \in \mathbb{N}$ the spectrum $\Sigma^{n} X$ is the suspension spectrum of a finite $\mathrm{CW}$-complex. Note that the finite spectra are precisely the compact objects in $\mathcal{C}$. Therefore $\mathcal{C}$ is a compactly generated triangulated category, since $\pi_{n}^{s}(X)=0$ for all $n \in \mathbb{Z}$ implies $X=0$. These facts are well known, see for instance [16].

4.1. $\mathfrak{p}$-localization. For every prime ideal $\mathfrak{p}$ in $\mathbb{Z}$ we denote by $X \rightarrow X_{\mathfrak{p}}$ the $\mathfrak{p}$ localization map for a spectrum $X$. The fibre of the rational localization $X \rightarrow X_{(0)}$ is denoted by $X_{\text {tor }}$.

Theorem 4.1. Let $X$ be an endofinite spectrum.

(1) The $\mathfrak{p}$-localization map $X \rightarrow X_{\mathfrak{p}}$ is a split epimorphism for every prime ideal $\mathfrak{p}$ in $\mathbb{Z}$.

(2) $X \cong X_{\text {tor }} \vee X_{(0)}$.

(3) Let $Y=X_{\text {tor. }}$. Then $Y \cong \bigvee_{\mathfrak{p}} Y_{\mathfrak{p}}$, where $\mathfrak{p}$ runs through all non-zero prime ideals in $\mathbb{Z}$.

Proof. (1) Fix a prime ideal $\mathfrak{p}$. The localization map $X \rightarrow X_{\mathfrak{p}}$ induces, by definition, for every $n \in \mathbb{Z}$ an isomorphism $\pi_{n}^{s}(X) \otimes_{\mathbb{Z}} \mathbb{Z}_{\mathfrak{p}} \cong \pi_{n}^{s}\left(X_{\mathfrak{p}}\right)$, which extends to an isomorphism

$$
\{C, X\} \otimes_{\mathbb{Z}} \mathbb{Z}_{\mathfrak{p}} \cong\left\{C, X_{\mathfrak{p}}\right\}
$$

for all $C$ in $\mathcal{C}_{0}$. Therefore $\operatorname{Ann} X \subseteq \operatorname{Ann} X_{\mathfrak{p}}$. If $X$ is indecomposable endofinite, then $X_{\mathfrak{p}}$ is a direct factor of a wedge of copies of $X$ by Corollary 3.7. Thus $X_{\mathfrak{p}}=X$ or $X_{\mathfrak{p}}=0$, and therefore $X \rightarrow X_{\mathfrak{p}}$ is a split epimorphism. An arbitrary endofinite spectrum $X$ is a wedge of indecomposable endofinite spectra by Proposition 3.3. and therefore $X \rightarrow X_{\mathfrak{p}}$ is a split epimorphism since $\mathfrak{p}$-localization preserves wedges; e.g. see [16, Corollary 8.3].

(2) The sequence $0 \rightarrow X_{\text {tor }} \rightarrow X \rightarrow X_{(0)} \rightarrow 0$ is split exact by (1).

(3) Suppose first that $Y$ is indecomposable. There exists a prime ideal $\mathfrak{q} \neq 0$ such that $\{C, Y\} \otimes_{\mathbb{Z}} \mathbb{Z}_{\mathfrak{q}} \neq 0$ for some $C$ in $\mathcal{C}_{0}$. Thus Ann $Y_{\mathfrak{q}} \neq \mathcal{C}_{0}$, and therefore $Y=Y_{\mathfrak{q}}$ by (1). Moreover, $Y_{\mathfrak{p}}=0$ for all $\mathfrak{p} \neq \mathfrak{q}$ since $Y$ is torsion, and $Y \cong \bigvee_{\mathfrak{p}} Y_{\mathfrak{p}}$ follows. Finally, the assertion holds for arbitrary $Y$ since $\mathfrak{p}$-localization preserves wedges. 
4.2. The endolength. Let $X$ be any spectrum. For every $n \in \mathbb{Z}$ we denote by $\ell_{n}(X)$ the length of the stable homotopy group $\pi_{n}^{s}(X)$ as $\{X, X\}$-module, and we call

$$
\ell(X)=\left(\ell_{n}(X)\right)_{n \in \mathbb{Z}}
$$

the endolength of $X$. The endolength leads to a nice characterisation of endofinite spectra:

Proposition 4.2. A spectrum $X$ is endofinite if and only if $\ell_{n}(X)$ is finite for all $n \in \mathbb{Z}$.

Proof. Consider the full subcategory $\mathcal{C}_{X}$ of spectra $Y$ such that $\left\{\Sigma^{n} Y, X\right\}$ is of finite length over $\{X, X\}$ for all $n \in \mathbb{Z}$. This is easily seen to be closed under cofibrations and retracts, and therefore $\mathcal{C}_{X}$ is a thick subcategory of $\mathcal{C}$. On the other hand, $\mathcal{C}_{0}$ is the smallest thick subcategory containing the sphere spectrum $S^{0}$; e.g. see [10] Theorem 2.1.3]. Consequently, if $S^{0}$ is an object of $\mathcal{C}_{X}$ then $\mathcal{C}_{0}$ is contained in $\mathcal{C}_{X}$. In particular, $X$ is endofinite.

We continue with another interpretation of the length $\ell_{n}(X)$. Let $\mathfrak{I}=\operatorname{Ann} X$ and denote by $\mathcal{S}=\mathcal{S}_{\mathfrak{I}}$ the corresponding Serre subcategory of $\mathcal{A}=\bmod \mathcal{C}_{0}$. The localizing subcategory of $\operatorname{Mod} \mathcal{C}_{0}$ generated by $\mathcal{S}$ is denoted by $\mathcal{T}$, and the quotient functor $Q: \operatorname{Mod} \mathcal{C}_{0} \rightarrow \operatorname{Mod} \mathcal{C}_{0} / \mathcal{T}$ identifies $\mathcal{A} / \mathcal{S}$ with a full subcategory of $\operatorname{Mod} \mathcal{C}_{0} / \mathcal{T}$; see [12, Theorem 2.6].

Lemma 4.3. Let $X$ be a pure-injective spectrum. Then $\ell_{n}(X)$ equals the length of $H_{S^{n}}$ in $\operatorname{Mod} \mathcal{C}_{0} / \mathcal{T}$, which is also the length of $H_{S^{n}}$ in $\mathcal{A} / \mathcal{S}$; in particular $\ell_{n}(X)=$ $\ell_{\mathfrak{I}}\left(S^{n}\right)$.

The proof of this lemma is essentially an application of the following easy lemma:

Lemma 4.4. Let $M$ and $N$ be objects in an abelian category and suppose that $N$ is an injective cogenerator. Then the length of $M$ equals the length of the $\operatorname{End}(N)$ module $\operatorname{Hom}(M, N)$.

Proof. Straightforward.

Proof of Lemma 4.3. Put $M=Q\left(H_{S^{n}}\right)$ and $N=Q\left(H_{X}\right)$, and observe that

$$
\{Y, X\} \cong \operatorname{Hom}\left(H_{Y}, H_{X}\right) \cong \operatorname{Hom}\left(Q\left(H_{Y}\right), Q\left(H_{X}\right)\right)
$$

for all $Y$ in $\mathcal{C}$ by Lemma 2.1. Also, $Q\left(H_{X}\right)$ is injective by the same lemma, since $X$ is pure-injective. The assertion now follows from the preceding lemma, since $\operatorname{Hom}\left(M^{\prime}, N\right) \neq 0$ for all $M^{\prime}=Q(A) \neq 0$ with $A \in \mathcal{A}$. Moreover, $M^{\prime}$ is a simple object in $\operatorname{Mod} \mathcal{C}_{0} / \mathcal{T}$ if and only if $M^{\prime}$ is simple when regarded as an object in $\mathcal{A} / \mathcal{S}$. Finally, $\ell_{n}(X)=\ell_{\mathfrak{I}}\left(S^{n}\right)$ follows from Lemma 1.11 .

We are now in a position to compute the endolength of a spectrum $X$ from a decomposition $X \cong \bigvee_{i} X_{i}$. We proceed in two steps.

Proposition 4.5. Let $X$ be a spectrum and $I$ be a non-empty set. Suppose that $\bigvee_{I} X$ is pure-injective. Then for all $n \in \mathbb{Z}$

$$
\ell_{n}\left(\bigvee_{I} X\right)=\ell_{n}(X)=\ell_{n}\left(\prod_{I} X\right)
$$

Proof. The assertion follows from Lemma 4.3, since $\operatorname{Ann}_{I} X=\operatorname{Ann} X=$ Ann $\prod_{I} X$ by Lemma 3.5 . 
Proposition 4.6. Let $X \cong \bigvee_{i \in I} X_{i}$ be a decomposition of a pure-injective spectrum. Suppose that any indecomposable spectrum arises as a wedge summand of $X_{i}$ for at most one $i \in I$. Then for all $n \in \mathbb{Z}$

$$
\ell_{n}\left(\bigvee_{i \in I} X_{i}\right)=\sum_{i \in I} \ell_{n}\left(X_{i}\right)
$$

Proof. We keep the above notation, and for every $i \in I$ we denote by $Q_{i}: \operatorname{Mod} \mathcal{C}_{0} / \mathcal{T}$ $\rightarrow \operatorname{Mod} \mathcal{C}_{0} / \mathcal{T}_{i}$ the quotient functor with respect to the localizing subcategory $\mathcal{T}_{i}$ which is generated by $\mathcal{S}_{\operatorname{Ann} X_{i}}$. Now suppose that $A \neq 0$ is any subquotient of $Q\left(H_{S^{n}}\right)$ in $\mathcal{A} / \mathcal{S}$. Then $Q_{i}(A) \neq 0$ for some $i \in I$, since

$$
0 \neq \operatorname{Hom}\left(A, Q\left(H_{X}\right)\right) \cong \coprod_{i} \operatorname{Hom}\left(A, Q\left(H_{X_{i}}\right)\right) \cong \coprod_{i} \operatorname{Hom}\left(Q_{i}(A), Q_{i} \circ Q\left(H_{X_{i}}\right)\right) .
$$

If $A$ is simple then $Q_{i}(A) \neq 0$ for precisely one $i \in I$ by our assumption on the family $\left(X_{i}\right)_{i \in I}$, since the injective envelope of $A$ in $\operatorname{Mod} \mathcal{C}_{0} / \mathcal{T}$ is isomorphic to $Q\left(H_{Y}\right)$ for some indecomposable summand $Y$ of $X$. Using the exactness of the $Q_{i}$, the formula for $\ell_{n}\left(\bigvee_{i \in I} X_{i}\right)$ follows from the fact that $\ell_{n}$ computes the length of $Q\left(H_{S^{n}}\right)$ by Lemma 4.3

Corollary 4.7. Let $X$ be an endofinite spectrum with $\mathfrak{I}=\operatorname{Ann} X$. Then for all $n \in \mathbb{Z}$

$$
\ell_{n}(X)=\sum_{\mathfrak{I} \subseteq \mathfrak{J} \in \operatorname{Max} \mathcal{C}_{0}} \ell_{n}\left(E_{\mathfrak{J}}\right)
$$

Proof. Combine Proposition 4.5 and Proposition 4.6 with the decomposition $X \cong$ $\bigvee_{\mathfrak{J}} X_{\mathfrak{J}}$ from Corollary 3.6

4.3. Subgroups of finite definition. Let $X$ be any spectrum. Given a finite spectrum $F$ and an element $\phi \in \pi_{n}^{s}(F)$ for some $n \in \mathbb{Z}$, we denote by $\pi_{n, \phi}^{s}(X)$ the image of the induced map

$$
\{\phi, X\}:\{F, X\} \longrightarrow\left\{S^{n}, X\right\}=\pi_{n}^{s}(X) .
$$

A subgroup of $\pi_{n}^{s}(X)$ which is of the form $\pi_{n, \phi}^{s}(X)$ for some $\phi \in \pi_{n}^{s}(F)$ is said to be of finite definition. Note that any subgroup of finite definition is automatically a $\{X, X\}$-submodule of $\pi_{n}^{s}(X)$.

Lemma 4.8. Let $\phi \in \pi_{n}^{s}(F)$ for some finite spectrum $F$.

(1) The subgroups of finite definition form a lattice in $\pi_{n}^{s}(X)$ for every spectrum $X$.

(2) If $\left(X_{i}\right)_{i \in I}$ is a family of spectra, then

$$
\pi_{n, \phi}^{s}\left(\bigvee_{i} X_{i}\right) \cong \coprod_{i} \pi_{n, \phi}^{s}\left(X_{i}\right)
$$

and

$$
\pi_{n, \phi}^{s}\left(\prod_{i} X_{i}\right) \cong \prod_{i} \pi_{n, \phi}^{s}\left(X_{i}\right)
$$

Proof. (1) Let $\phi_{i} \in \pi_{n}^{s}\left(F_{i}\right)(i=1,2)$. Then $\pi_{n, \phi_{1}}^{s}(X)+\pi_{n, \phi_{2}}^{s}(X)=\pi_{n, \phi}^{s}(X)$, where $\phi: S^{n} \rightarrow F_{1} \vee F_{2}$ has components $\phi_{i}$. Let $\psi: F_{1} \vee F_{2} \rightarrow G$ be a cofibre of $\phi$, with components $\psi_{i}$, and set $\chi=\psi_{1} \phi_{1}$. The sequence $\{G, X\} \rightarrow\left\{F_{1} \vee F_{2}, X\right\} \rightarrow\left\{S^{n}, X\right\}$ is exact, and it follows that $\pi_{n, \phi_{1}}^{s}(X) \cap \pi_{n, \phi_{2}}^{s}(X)=\pi_{n, \chi}^{s}(X)$.

(2) Clear, since $\{\phi,-\}$ preserves wedges and products. 
The endofiniteness of a spectrum can be characterized in terms of the lattice of subgroups of finite definition.

Proposition 4.9. A spectrum $X$ is endofinite if and only if $\pi_{n}^{s}(X)$ has the ascending and descending chain conditions on subgroups of finite definition for all $n \in \mathbb{Z}$. If this holds, then the $\{X, X\}$-submodules of $\pi_{n}^{s}(X)$ are precisely the subgroups of finite definition.

Proof. As for modules; see [3, Proposition 4.1].

For the sake of completeness we include the following characterization of the spectra having the descending chain condition on subgroups of finite definition:

Theorem 4.10. The following conditions are equivalent for a spectrum $X$ :

(1) $X$ is $\Sigma$-pure-injective, i.e. $\bigvee_{I} X$ is pure-injective for every set I;

(2) the canonical map $\bigvee_{\mathbb{N}} X \rightarrow \prod_{\mathbb{N}} X$ is a split monomorphism;

(3) the descending chain condition holds for the subgroups of finite definition of $\pi_{n}^{s}(X)$ for every $n \in \mathbb{Z}$

(4) every product of copies of $X$ is a wedge of indecomposable spectra with local endomorphism rings.

Proof. Adapt the argument for modules [11], using the restricted Yoneda functor $\mathcal{C} \rightarrow \operatorname{Mod} \mathcal{C}_{0}$.

Remark 4.11. Concepts and results from this section carry over to any compactly generated triangulated category $\mathcal{C}$ if one replaces the sphere spectrum $S$ by any set of compact objects which generates $\mathcal{C}$.

\section{A CRITERION FOR ENDOFINITENESS}

In this section we give a criterion for the suspension spectrum of a $\mathrm{CW}$-complex to be endofinite in terms of its singular homology groups. Following the definition of an endofinite module [3], we say that a CW-complex is endofinite if the stable homotopy groups $\pi_{n}^{s}(X)$ are modules of finite length over $\{X, X\}$ for all $n \in \mathbb{Z}$.

Theorem 5.1. Let $X$ be a connected $C W$-complex such that the singular homology groups $H_{n}(X ; \mathbb{Z})$ are finite for all $n>0$. Then $X$ is an endofinite $C W$-complex, and therefore its suspension spectrum is also endofinite.

Proof. We prove that a CW-complex $X$ satisfying the above assumptions has finite stable homotopy groups, i.e. $\pi_{n}^{s}(X)$ is finite for all $n \in \mathbb{Z}$. Clearly, this implies that $X$ is an endofinite CW-complex, and therefore the suspension spectrum of $X$ is endofinite by Proposition 4.2 To prove the finiteness of the stable homotopy groups, we apply the Atiyah-Hirzebruch-Whitehead spectral sequence for the (unreduced) homology theory $h_{n}(-)=\pi_{n}^{s}\left(-_{+}\right)$. The subscript + means adding of a disjoint basepoint. The coefficient groups $h_{n}$ (pt.) are the stable homotopy groups of $S^{0}$ and are known to be trivial for $n<0$, isomorphic to $\mathbb{Z}$ for $n=0$, and finite for $n>0$. Therefore, the $E^{2}$-term of the spectral sequence, $E_{p, q}^{2}=H_{p}\left(X ; h_{q}(\right.$ pt. $\left.)\right)$, has non-trivial values only for $p, q \geq 0$, and these are finite with the sole exception $E_{0,0}^{2}=\mathbb{Z}$. This form is preserved for all $E^{k}$-terms, $k \geq 2$.

The spectral sequence converges to $h_{*}(X)$. This means that $h_{n}(X)$ is the limit of groups $F_{0, n} \subseteq F_{1, n-1} \subseteq \ldots$ which are inductively calculated from $F_{0, n}=E_{0, n}^{\infty}$ and the extension problem $0 \rightarrow F_{p-1, n-p+1} \rightarrow F_{p, n-p} \rightarrow E_{p, n-p}^{\infty} \rightarrow 0$. From this 
inductive construction it follows easily that $F_{p, n-p}=0$ for $n<0$ and $h_{n}(X)=F_{n, 0}$ is finite for $n>0$. For the respective reduced homology theory $\tilde{h}_{n}(-)=\pi_{n}^{s}(-)$ we get

$$
\pi_{n}^{s}(X)=\pi_{n}^{s}\left(X_{+}\right) / \pi_{n}^{s}\left(S^{0}\right)= \begin{cases}0 & \text { for } n \leq 0 \\ \text { finite } & \text { for } n>0\end{cases}
$$

Important examples of CW-complexes with finite homology are the classifying spaces of finite groups.

Corollary 5.2. The classifying space $B G$ of a finite group $G$ is endofinite.

Proof. Since the homology groups $H_{n}(B G ; \mathbb{Z})$ are finite in all positive degrees, we may apply the preceding theorem.

In Theorem [5.1, the condition that $X$ be connected cannot be omitted. For example, $X=S^{0}$ has finite homology in all positive degrees but the $\{X, X\}$-module $\pi_{0}^{s}(X) \cong \mathbb{Z}$ is not of finite length.

It has been pointed out by Stefan Schwede that Theorem5.1 can be generalized as follows.

Theorem 5.3. A connected $C W$-spectrum $X$ is endofinite if and only if the homology groups $H_{n}(X ; \mathbb{Z})$ with coefficients in $\mathbb{Z}$ are modules of finite length over $\{X, X\}$ for all $n \in \mathbb{Z}$.

Proof. The argument is essentialy that given in the proof of Theorem 5.1 To prove endofiniteness of $X$ one uses the Atiyah-Hirzebruch-Whitehead spectral sequence

$$
H_{*}\left(X ; \pi_{*}^{s}\right) \Longrightarrow \pi_{*}^{s}(X)
$$

as above. To prove finiteness of the homology of $X$ one uses the less well-known spectral sequence

$$
\operatorname{Tor}^{\pi_{*}^{s}}\left(\pi_{*}^{s}(X), \mathbb{Z}\right) \Longrightarrow H_{*}(X ; \mathbb{Z}) .
$$

Both spectral sequences are natural in $X$ and can be found in [5].

\section{ACKNOWLEDGEMENT}

Both authors would like to thank Stefan Bauer and Hans-Werner Henn for a number of helpful conversations concerning the material of this paper. The second named author was supported by the SFB 343.

\section{REFERENCES}

[1] J.F. Adams, Stable homotopy and generalised homology, The University of Chicago Press (1974). MR 53:6534

[2] G. Carlsson, Equivariant stable homotopy and Segal's Burnside ring conjecture, Ann. Math. 120 (1984), 189-224. MR 86f:57036

[3] W.W. Crawley-Boevey, Modules of finite length over their endomorphism ring, in: Representations of algebras and related topics, eds. S. Brenner and H. Tachikawa, London Math. Soc. Lec. Note Series 168 (1992), 127-184. MR 94h:16018

[4] W.W. Crawley-Boevey, Locally finitely presented additive categories, Comm. Algebra 22 (1994), 1641-1674. MR 95h:18009

[5] A.D. Elmendorf and I. Kriz and M.A. Mandell and J.P. May, Rings, modules, and algebras in stable homotopy theory, Amer. Math. Soc., Mathematical Surveys and Monographs 47 (1997). MR 97h:55006 
[6] P. Freyd, Stable homotopy, in: Proceedings of the conference on categorical algebra, La Jolla, Springer-Verlag, New York (1966), 121-172. MR 35:2280

[7] P. Gabriel, Des catégories abéliennes, Bull. Soc. Math. France 90 (1962), 323-448. MR 38:1144

[8] L. Gruson and C.U. Jensen, Deux applications de la notion de L-dimension, C. R. Acad. Sci. Paris Ser. A 282 (1976), 23-24. MR 53:5706

[9] H.-W. Henn, Finiteness properties of injective resolutions of certain unstable modules over the Steenrod algebra and applications, Math. Ann. 291 (1991), 191-203. MR 92i:55016

[10] M. Hovey, J.H. Palmieri, N.P. Strickland, Axiomatic stable homotopy theory, Mem. Amer. Math. Soc. 610 (1997). MR 98a:55017

[11] C.U. Jensen and H. Lenzing, Model theoretic algebra, Gordon and Breach, New York (1989). MR 91m:03038

[12] H. Krause, The spectrum of a locally coherent category, J. Pure Appl. Algebra 114 (1997), 259-271. MR 98e:18006

[13] H. Krause, Exactly definable categories, J. Algebra 201 (1998), 456-492. MR 99j:18010

[14] H. Krause, Smashing subcategories and the telescope conjecture - an algebraic approach, Invent. Math. 139 (2000), 99-133. CMP 2000:06

[15] H. Krause, Decomposing thick subcategories of the stable module category, Math. Ann. 313 (1999), 95-108. CMP 99:07

[16] H.R. Margolis, Spectra and the Steenrod algebra, North-Holland (1983). MR 86j:55001

[17] A. Neeman, The Grothendieck duality theorem via Bousfield's techniques and Brown representability, J. Amer. Math. Soc. 9 (1996), 205-236. MR 96c:18006

[18] J.L. Verdier, Catégories derivées, état 0, SGA 4 $\frac{1}{2}$, Springer Lec. Notes 569 (1977), 262-311. MR 57:3132

Fakultät für Mathematik, Universität Bielefeld, 33501 Bielefeld, Germany

E-mail address: henning@mathematik.uni-bielefeld.de

Fakultät für Mathematik, Universität Bielefeld, 33501 Bielefeld, Germany

E-mail address: reichenb@mathematik.uni-bielefeld.de 